\title{
INTRODUCTION TO THE SPECIAL ISSUE ON RELIABILITY AND SECURITY OF EHEALTH INFORMATION SYSTEMS
}

With the increasing population and aging society in several countries, healthcare providers aim to enhance the quality of the healthcare services while balancing the risk mitigation and service cost. Therefore, several new information technologies and innovative communication methodologies have evolved to improve the healthcare sector. ICT-based technologies help in decreasing the healthcare system overhead and increasing the quality of healthcare services. These technologies may include biosensors, computer aided diagnosis, Wireless Body Sensor Network (WBSN), mobile health, Radio Frequency Identification (RFID), cloud computing, communication protocols, electronic medical records, big data, and internet of things (IoT). Therefore, the complexity of healthcare systems has increased dramatically during the last two decades. Despite having several approaches developed for testing and verification of healthcare systems, ICT related medical incidents that led into losses of money, time, reputation, and in certain cases, lives, still happen frequently. It is believed that healthcare systems do not get enough testing and verification before being put into use, even though they are considered safety critical systems. This is due to the high cost of testing, short time to marker, and the lack of proper testing and verification techniques in the literature. Design errors, system usage problems, design reliability issues, compliance Issues, system failure and vulnerabilities in eHealth care system can lead to critical conditions or even death.

The special issue publishes three papers that extended from papers presented at IEEE Healthcom 2015, in addition to two new submitted papers. The first paper in this issue by Pervez et al. titled "Improvement Strategies for Device Interoperability Middleware (DIM) using Formal Reliability analysis", where the authors used probabilistic model checker PRISM for analyzing Device Interoperability Middleware (DIM) [1]. The second paper by Bhardwaj and Prasad is titled "PRAVAH: Parameterised Information Flow Control in e-Health". The authors addressed the problem of enforcing information flow control (IFC) in hospital domains in eHealth systems using a parameterised lattice-based IFC framework called PRAVAH [2]. The third paper by Ayache et al. is titled "Analysis and verification of XACML policies in a medical cloud environment". The authors presented a Cloud Policy Verification Service (CPVS) for the analysis and the verification of access control policies specified using XACML [3]. The fourth paper by Sadki and Bakkali is titled "Resolving conflicting privacy policies in m-health based on prioritization". The authors presented resent an approach to resolve the problem of conflicting privacy policies in mobile health environments using AHP (Analytic Hierarchy Process) prioritization technique and reputation mechanism [4]. The fifth paper by Gawanmeh et al. is titled "Formal Analysis of a Microfluidic Device for Blood Cell Separation". The authors used formal analysis in order to formalize and validate the movement of blood cells in a microdevice under different forces for the purpose of cell separation [5]. We would like to thank the editorial board of SCPE for the efforts they made to make this special issue, and all the reviewers for their efforts and feedback.

Kashif Saleem, King Saud University, Saudi Arabia

Amjad Gawanmeh, Concordia University, Montreal, Canada and Khalifa University, UAE

\section{REFERENCES}

[1] Usman Pervez, Asiah Mahmood, Osman Hasan, Khalid Latif, and Amjad Gawanmeh, , and Ahmad Alomari. "Improvement Strategies for Device Interoperability Middleware (DIM) using Formal Reliability Analysis." Scalable Computing: Practice and Experience 17 (3), 2016, 150-170.

[2] Chandrika Bhardwaj and Sanjiva Prasad. "PRAVAH: Parameterised Information Flow Control in e-Health." Scalable Computing: Practice and Experience 17 (3), 2016, 171-187.

[3] Meryeme Ayache, Mohammed Erradi, Ahmed Khoumsi, and Bernd Freisleben. "Analysis and Verification of XACML Policies in a Medical Cloud Environment." Scalable Computing: Practice and Experience 17 (3), 2016, 189-205.

[4] Souad Sadki, Hanan EL Bakkali. "Resolving conflicting privacy policies in m-health based on prioritization." Scalable Computing: Practice and Experience 17 (3), 2016, 207-226.

[5] Amjad Gawanmeh, Anas Alazzam, and Bobby Mathew. "Formal Verification of a Microfluidic Device for Blood Cell Separation." Scalable Computing: Practice and Experience 17 (3), 2016, 227-235. 